\title{
Neoantigens and genome instability: impact on immunogenomic phenotypes and immunotherapy response
}

\author{
Elaine R. Mardis
}

\begin{abstract}
The resurgence of immune therapies in cancer medicine has elicited a corresponding interest in understanding the basis of patient response or resistance to these treatments. One aspect of patient response clearly lies in the genomic alterations that are associated with cancer onset and progression, including those that contribute to genomic instability and the resulting creation of novel peptide sequences that may present as neoantigens. The immune reaction to these unique 'non-self' peptides is frequently suppressed by the tumor itself, but the use of checkpoint blockade therapies, personalized vaccines, or a combination of these treatments may elicit a tumorspecific immune response that results in cell death. Massively parallel sequencing, coupled with different computational analyses, provides unbiased identification of the germline and somatic alterations that drive cancer development, and of those alterations that lead to neoantigens. These range from simple point mutations that change single amino acids to complex alterations, such as frameshift insertion or deletion mutations, splice-site alterations that lead to exon skipping, structural alterations that lead to the formation of fusion proteins, and other forms of collateral damage caused by genome instability that result in new protein sequences unique to the cancer. The various genome instability phenotypes can be identified as alterations that impact DNA replication or mismatch repair pathways or by their genomic signatures. This review provides an overview of current knowledge regarding the fundamentals of genome replication and of both germline and somatic alterations that disrupt normal replication, leading to various forms of genomic instability in cancers, to the resulting generation of neoantigens and, ultimately, to immune-responsive and resistant phenotypes.
\end{abstract}

\section{Background}

The fidelity with which our genome is copied prior to cell division is remarkable in its consistency over time. This consistency results from a variety of enzymatic DNA replication, proofreading, and damage repair functions that work in concert to minimize alterations from one cell division to the next. Nevertheless, these highfidelity processes can become compromised by a variety of genomic alterations that subsequently result in the development of cancer, in which the normal genomewide mutation rate becomes accelerated. Often, this consequence is due to inherited or de novo alterations in the germline that impact the proper function of enzymes that are involved in these processes, leading to different

Correspondence: Elaine.Mardis@nationwidechildrens.org

Institute for Genomic Medicine at Nationwide Children's Hospital, The Ohio State University College of Medicine, Children's Drive, Colombus, $\mathrm{OH} 43205$, USA

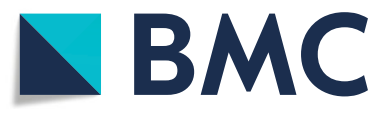

(C) The Author(s). 2019 Open Access This article is distributed under the terms of the Creative Commons Attribution 4.0 International License (http://creativecommons.org/licenses/by/4.0/), which permits unrestricted use, distribution, and

reproduction in any medium, provided you give appropriate credit to the original author(s) and the source, provide a link to the Creative Commons license, and indicate if changes were made. The Creative Commons Public Domain Dedication waiver (http://creativecommons.org/publicdomain/zero/1.0/) applies to the data made available in this article, unless otherwise stated. manifestations of genome instability. Because the enzymatic functions that normally ensure genome replication fidelity are altered, the resulting errors can lead to secondary, somatic alterations of several types that may change protein-coding sequences in the genome. When alterations occur in cancer-related genes, a progression to malignancy results. Alternatively, mutations may occur in so-called 'passenger genes' that have no link to cancer onset or progression. In either case, the alterations that have resulted (directly or indirectly) from genomic instability in genes that are transcribed and translated, encode novel peptide sequences that are unique to the cancer cell. During normal protein degradation, these novel peptides may be bound by major histocompatibility complex (MHC) proteins that present them on the cell surface as 'neoantigens' (i.e., tumorspecific peptides that can be recognized by the immune 
system as non-self, making the cancer cells targets for destruction). This process is summarized in Fig. 1.

Many years of basic cancer immunology research have delineated the mechanisms by which cancer cells suppress this anti-cancer immune response through tolerance and immune suppression mechanisms. However, immune-based cancer therapies ('immunotherapies') such as checkpoint blockade inhibitors, which were inspired in their formulation by the research that revealed immune checkpoint suppression, have now established that the therapeutic (antibody-based) inhibition of immune suppression checkpoint proteins allows the immune system to become engaged and to eradicate the cancer cells. Hence, this review will examine knowledge accrued to date that links genome instability (in its many forms) to the generation of neoantigens and to treatment response or resistance to different immunotherapies. Taken together, this review explores how genomic instability and its consequences are emerging as a key clinical consideration in cancer precision medicine.

\section{Fundamentals of genome replication}

The human genome is large and repetitive, yet each human cell division is accompanied by the highly accurate replication of approximately six billion base-pairs of DNA. Fidelity in replication is a critical component of this process, and both polymerase proofreading by polymerase epsilon and delta and the mismatch repair
(MMR) system improve basic replication fidelity by about 100-fold [1-3]. In normal chromosomal replication processes, replication errors inevitably escape correction and provide a baseline rate of somatic mutations, which accumulate in the resulting cell lineage over time and with increasing age. When these fundamental aspects of replication fidelity are disrupted by functional alterations in MMR system enzymes or in the proofreading polymerases, as well as in the settings of other base excision and homologous repair defects, the baseline mutation rate at genome replication in the context of cell division is elevated to differing degrees [4], and genomic instability results. Such cancer susceptibility defects can be acquired by individuals through either inheritance or spontaneous mutation. Historically, the diagnosis of conditions that involve genomic instability, such as Lynch syndrome or the presence of BRCA1/2 or xeroderma pigmentosum (XP) defects, has involved the use of imaging-based cancer screening at an earlier age and with increased frequency than that appropriate for the general population. Other assays, such as colonoscopy, are also used to detect early-onset cancers. In the present day, however, the different types of genomic instability imparted by replication defects-including mismatch repair, base excision, and homologous end joining-increasingly have implications for cancer treatment and for treatment response, as this review explores. Further resolution to the nuanced impact of

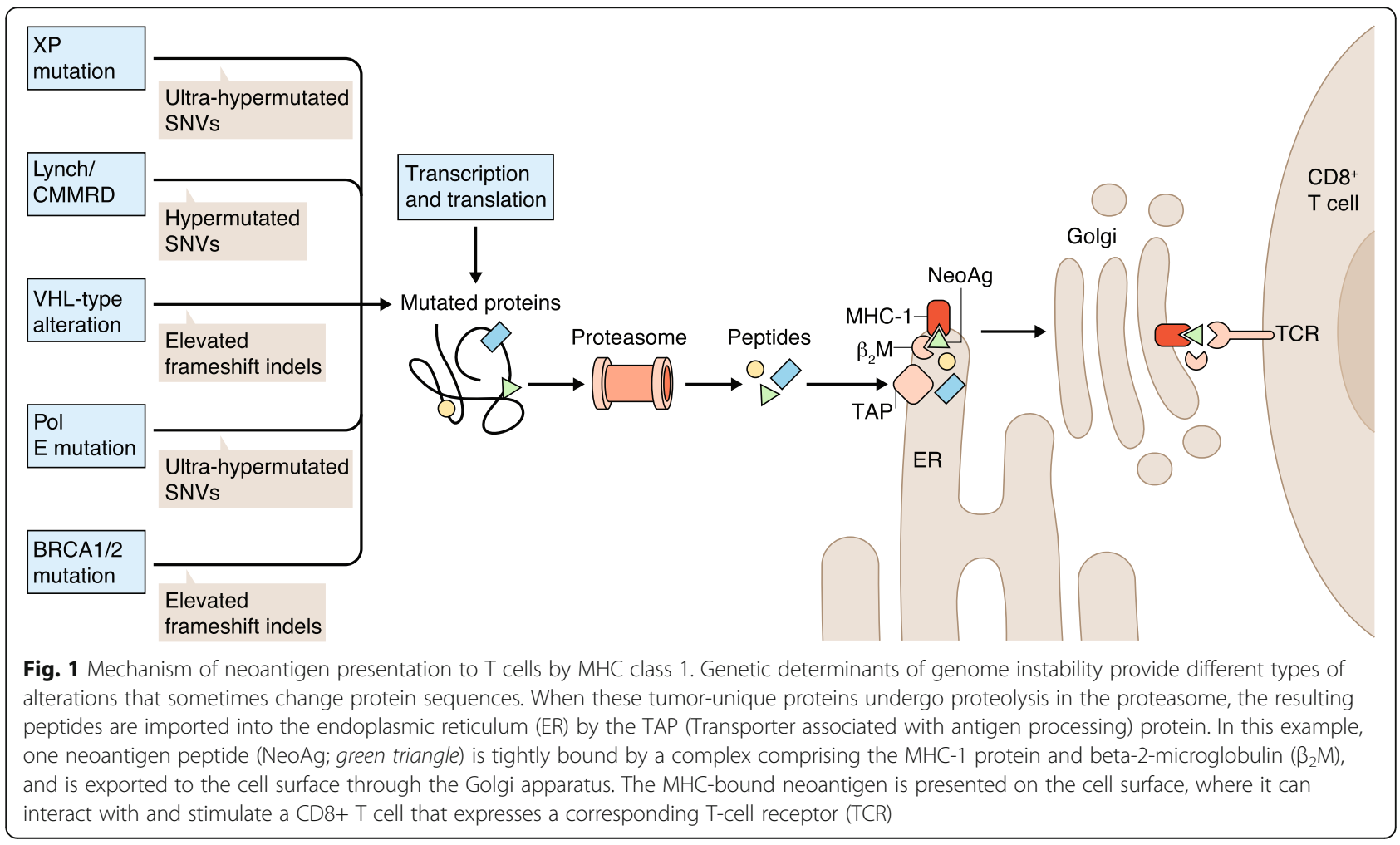


genomic instability is also emerging, as we realize that different genomic alterations elicit different responses to immune checkpoint blockade therapies.

\section{Germline and somatic contributors to genomic instability} Basic research to characterize the enzymatic machinery of DNA replication [5] and to define genetic syndromes that result from fidelity defects in DNA replication [2] has helped to elucidate the fundamental processes involved in eukaryotic chromosomal replication, to identify the enzymes responsible for replication fidelity and their variants, and to develop assays that diagnose these syndromes [6]. The specific details of DNA replication fidelity mechanisms and their associated defects are beyond the scope of this review, but numerous genes and their pathogenic alterations have been catalogued in terms of their contribution to genomic instability. In general, these genes and mutations can be altered in the germline (by either inherited or de novo mechanisms), in the somatic tissue genome, or in both. Inherited mismatch repair defects can be seen in Lynch syndrome, which is characterized by autosomal dominant inheritance of heterozygous pathogenic germline mutations in one of the MMR genes (MLH1, MSH2, MSH6, or PMS2) [7]; in Lynch-like syndrome, which results from double somatic mutations in one of the MMR genes; and in constitutional MMR deficiency syndrome (CMMRD), an autosomal recessive disorder caused by biallelic pathogenic germline mutations in MMR genes [8].

Lynch syndrome is the most prevalent of these mismatch repair defects at an estimated 1 in 279 individuals [9], although Lynch-like syndrome has recently been estimated to have a similar incidence [10]. The proportions of mutations in each of the Lynch syndrome genes are MLH1 (40\%), MSH2 (34\%), MSH6 (18\%), and PMS2 (2\%), with the cancer risk varying depending on the gene involved. Sporadic MMR deficiency also occurs, typically as the result of hypermethylation of the MLH1 promoter, which causes loss of MLH1 protein expression [11]. This sporadic form of MMR deficiency is a common driver of colorectal and endometrial cancers, identified in 69 and $94 \%$ of $M L H 1$ and PMS2 non-mutated cases, respectively. Germline pathogenic mutations in POLD1 and POLE are found in the exonuclease domain and have been documented in familial cancer syndromes [12-19], although they occur at quite low population frequencies $(\leq 0.002)$. BRCA1, BRCA2, and PALB2 proteins are components of the protein complex that effects DNA repair at double-stranded breaks (DSBs), and alterations to the genes that encode these proteins have been linked to inherited breast, ovarian, and endometrial cancer susceptibility [20-25]. The incidence of BRCA1/2 inherited mutations has been estimated at 1 in 400 but this is subject to ancestry considerations, as has been well documented in certain populations. Similarly, these genes also can be mutated in the somatic genome and contribute to an overall increase in insertion and deletion mutations genome-wide.

In summary, genetic contributions to genome instability are inherited, sporadic, and somatic in nature, often combining to drive the development of cancer with a variety of impacts upon genome instability that are detectable by a variety of diagnostic approaches. Identifying these alterations has meaning in the contexts of cancer predisposition, monitoring, and early detection, as well as for indicating newer types of cancer therapy that can engage each patient's immune system in eradicating the disease.

\section{Approaches for the detection and diagnosis of genomic instability}

Historically, MMR defects have been diagnosed using a combination of PCR and the sequencing of specific microsatellite regions to detect microsatellite instability (MSI) and immunohistochemistry to assess the altered expression levels of MMR proteins within the tumor that might correlate with a diagnosis of high MSI. These assays were based on the understanding that defective mismatch repair leads to uncorrected DNA polymerase errors at mono- and dinucleotide microsatellite loci during genome replication, and could be correlated to methylation-based epigenetic silencing of MMR genes that leads to reduced levels of the encoded proteins. Although these assays were once considered adequate for the diagnosis of mismatch repair defects, recent largescale studies, aimed at characterizing somatic and germline alterations in tumor vs normal comparisons by the use of massively parallel or next generation sequencing (NGS), have led to an enriched understanding of the numbers and types of alterations that occur in genes associated with genome instability. In particular, NGSbased assays to detect and diagnose genomic instability are achieving increased resolution relative to previous approaches. Hence, our understanding of the consequences of genomic instability, with regard to how they may engage the immune system and determine responses to new immune therapy modalities, is described herein.

These NGS-based studies of tumor and germline DNA have illustrated that genes encoding replication, proofreading, or DSB repair proteins are concurrently altered on both alleles by loss of heterozygosity, monoallelic deletion, epigenetic silencing, or mutation in tumor tissue, thereby acting in concert with the inherited defect [26]. Moreover, certain types of genomic instability impart a specific sequence-based mutational signature that can be detected by appropriate computational analysis of NGS data $[27,28]$. For example, Nik-Zainal's group has used 
the specific mutational signatures from NGS whole genome sequencing of breast cancers to detect homologous repair defects in BRCA-complex-mediated genomic instability that predict patients who are likely to respond to poly ADP ribose polymerase (PARP) inhibitor therapy [29].

Beyond detecting specific genomic alterations, the accompanying mutational load or tumor mutational burden (TMB) can be quantified using somatic analysis pipelines $[26,30]$. The important roles played by proteins that are involved in maintaining proper DNA replication fidelity or DSB repair dictate that cancers with defective replication/proofreading or DSB repair have an elevated mutation rate when compared to cancers from the same tissue site without alterations to these proteins [27]. As these alterations, together with the normal stochastic background mutations that occur over time, impact protein-coding genes in the cancer genome and may change amino acid sequences, the resulting changes are referred to as the 'mutanome'. In particular, somatic alterations that change amino acid sequences create unique proteins that may, upon intracellular degradation, be bound by MHC molecules that present them on the cell surface, as discussed earlier. The interaction between $\mathrm{CD} 8+\mathrm{T}$ cells and $\mathrm{MHC}$ presented neoantigens elicits $\mathrm{T}$-cell-specific recognition of each 'non-self' neoantigen peptide, thereby allowing the patient's immune system to distinguish cancerous from normal cells. Neoantigens result from somatic changes, including simple point mutations that substitute a different amino acid, insertions or deletions of nucleotides that shift the open reading frame, and inversions, translocations, or other structural alterations that result in protein fusions.

Therefore, the NGS-based evaluation of cancers using analytical approaches that are capable of detecting these types of alterations both extends and refines the information available from the conventional immunohistochemistry (IHC), PCR and sequencing, or microarraybased chromosomal instability (CIN) assays that are used in the clinical diagnosis of genomic instability (Table 1). In effect, a broad-based NGS assay (exome or whole genome) provides greater resolution of the underlying germline and somatic defects and identifies the genomic consequences (the mutanome) that results from these defects, obviating the need for multiple assays to elucidate the underlying cause of each type of defect (methylation changes, mismatch repair defect (MMRd), or DSB repair). Beyond the diagnostic assay of genome instability, the identification of the tumor-unique neoantigens that are created by various defects in replication fidelity is emerging as an important therapeutic indication, both for predicting likely response to checkpoint blockade therapy and for designing personalized vaccines.

Table 1 Comparison of different assays used to detect mismatch repair defects and other predictors of immune therapy response or resistance

\begin{tabular}{|c|c|c|c|}
\hline Technique or assay & Description & Attributes & Deficiencies \\
\hline MSI-PCR & $\begin{array}{l}\text { PCR-based amplification of known } \\
\text { microsatellite loci, gel electrophoresis, } \\
\text { and software scoring to detect instability } \\
\text { as a multiple-band pattern in amplicons }\end{array}$ & $\begin{array}{l}\text { Focused test with rapid turn-around } \\
\text { time }\end{array}$ & $\begin{array}{l}\text { Interpretation difficulties, limited } \\
\text { to MSI diagnosis, no information } \\
\text { on genetic source of MMRd }\end{array}$ \\
\hline $\mathrm{dMMR} / \mathrm{IHC}$ & $\begin{array}{l}\text { Antibody-based staining of FFPE sections } \\
\text { from tumor, followed by microscopic } \\
\text { examination and scoring to detect MMR } \\
\text { proteins (MSH2, MSH6, MLH1, PMS2) }\end{array}$ & $\begin{array}{l}\text { Focused test using a conventional } \\
\text { pathology approach, inexpensive, } \\
\text { rapid turn-around time }\end{array}$ & $\begin{array}{l}\text { Evaluates the end result of MMR } \\
\text { protein depleting alterations only, } \\
\text { subject to inter-individual } \\
\text { interpretation }\end{array}$ \\
\hline $\mathrm{CIN} / \mathrm{FISH}$ & $\begin{array}{l}\text { Hybridization of centromere-specific } \\
\text { fluorescent probes to chromosomal } \\
\text { spreads, microscopic scoring of } \\
\text { centromeric counts to detect aneuploidy }\end{array}$ & $\begin{array}{l}\text { Genome-wide evaluation of } \\
\text { chromosomal instability }\end{array}$ & $\begin{array}{l}\text { Counting-based evaluation that } \\
\text { is subject to inter-individual } \\
\text { interpretation variability }\end{array}$ \\
\hline MMR/MSI-NGS panel & $\begin{array}{l}\text { NGS of genes for MMR proteins, mutation } \\
\text { detection and annotation of pathogenic } \\
\text { variants }\end{array}$ & $\begin{array}{l}\text { Focused evaluation of mutations } \\
\text { across known MMRd genes }\end{array}$ & $\begin{array}{l}\text { Insensitive to large-scale } \\
\text { alterations such as CNVs, } \\
\text { insufficient breadth for TMB or } \\
\text { neoantigen prediction }\end{array}$ \\
\hline NGS/WES & $\begin{array}{l}\text { NGS of all known coding exons of genes, } \\
\text { mutation detection and annotation of } \\
\text { pathogenic variants in genes }\end{array}$ & $\begin{array}{l}\text { Unbiased evaluation of mutations } \\
\text { across all coding genes, MSI } \\
\text { evaluation (added probes), neoantigen } \\
\text { prediction, TMB enabled }\end{array}$ & $\begin{array}{l}\text { Mutational signature calculation } \\
\text { may be compromised by lack } \\
\text { of breadth }\end{array}$ \\
\hline NGS/WGS & $\begin{array}{l}\text { NGS of whole genome libraries from } \\
\text { cancer and matched normal DNA, } \\
\text { comprehensive variant detection, and } \\
\text { annotation of variants }\end{array}$ & $\begin{array}{l}\text { Unbiased evaluation of mutations } \\
\text { across all coding genes, MSI } \\
\text { evaluation, neoantigen prediction, } \\
\text { TMB, mutational signature enabled }\end{array}$ & $\begin{array}{l}\text { Expensive to generate sufficient } \\
\text { coverage from low cellularity } \\
\text { tumors }\end{array}$ \\
\hline
\end{tabular}




\section{Genomic instability and neoantigen generation Neoantigen prediction}

The use of NGS data and computational analyses to identify genomic alterations in the DNA of cancers has been reviewed elsewhere [31]. These approaches now constitute the first step in identifying which alterations change the amino acid sequences of the encoded proteins, possibly resulting (following intracellular proteolytic degradation) in the production of novel peptides that have a strong predicted differential binding affinity for MHC molecules. The transition from detecting alterations to predicting neoantigens is typically accomplished by a series of computational steps that produce in silico tiled peptide sequences around the altered amino acid sequence of each novel peptide predicted in the mutanome. Similarly, NGS data are evaluated to type the MHC proteins that are encoded by each patient's germline, using specialized data analysis approaches that are necessitated by the hypervariable nature of these loci $[32,33]$. The process by which each novel peptide is evaluated for MHC-binding strength uses one of several published methods, each of which calculates the binding affinity of each novel peptide in the context of the human leukocyte antigen (HLA) proteins for that patient (using a neural net or other machine learning-based predictor) and compares it to the binding affinity of the native peptide. Following these calculations, filtering of the list of putative neoantigens uses RNA expression data from the tumor to identify which of the proposed strong binding peptides are expressed by the tumor. Filtering exome data eliminates potential false-positive results that are caused by a lack of NGS data coverage of the normal sample, or other contributors to false positivity [34]. A multitude of nuances are associated with the identification of potential neoantigens from NGS data and several pipelines have been developed to facilitate these analyses [34-38].

Hence, neoantigen prediction from NGS data produces two potentially valuable types of information: (i) the numbers and classes of neoantigens (e.g., MHC class 1 and/or class 2 binders); and (ii) the peptide sequences that could potentially be used in personalized vaccines aimed at eliciting neoantigen-specific T-cell responses [39]. Predictably, frameshift insertions or deletions [40, 41], exon skipping events, and protein fusions [42-44], although certainly more rare than point mutations, produce significantly altered peptides, which often have higher predicted affinity for MHC molecules than peptides that contain amino acidsubstitution mutations. Neoantigens that are derived from 'noncoding' sequences in the genome have also been reported; these are predominantly non-mutated, aberrantly expressed transcripts such as endogenous retroelements [45]. One commonly cited critique of computational approaches to neoantigen prediction is the high false-positive rate and the concern that these in silico predictions are missing important neoantigens. Several groups are attempting to address these challenges by adding mass-spectrometry-based evaluation of isolated MHC proteins from cancer samples that provide an inventory of peptides bound to MHC. These data are then compared to the corresponding computationally predicted neoantigens to differentiate true-positive from false-positive predictions. Over time and with increasing data of this type, such a dataset could be used to train a machine-learning-based algorithm to further refine in silico predictions prior to the use of neoantigens in a personalized vaccine approach [46-48].

\section{Immunotherapeutics and neoantigens}

The connection between genomic instability and neoantigen generation is pertinent to therapeutic cancer treatments known as immune checkpoint blockade inhibitor therapies. These antibody-based therapies were the products of basic cancer immunology studies carried out in the 1990s and early 2000s that identified mechanisms, based on immune checkpoints, by which tumors evade targeting and elimination by the host immune system $[49,50]$. Checkpoint proteins are typically involved in immune tolerance, preventing indiscriminate immune system attack, but several inhibitory immune checkpoint proteins that are expressed by cancer cells promote immune tolerance and permit tumor growth. Targeting these proteins with antibody-based drugs can remove the immune tolerance and permit $\mathrm{T}$-cell targeting, resulting in cancer cell death $[51,52]$. A general principle is that the greater the number of mutations or neoantigens present, the more likely it is that responses will be elicited from multiple, tumor-specific, T-cell populations in the context of checkpoint blockade therapy. This general principle has been somewhat borne out in clinical trials of different checkpoint blockade inhibitors, although it is certainly the case that some patients with low tumor mutational burden (and few neoantigens) also have responded to this type of treatment with tumor regression.

Several early clinical trials of immune checkpoint blockade inhibitors that were conducted in typically high mutational load tumors (such as melanoma and lung adenocarcinoma) used correlative genomic studies of tumor tissue from enrolled patients to identify a link between high TMB (>10 mutations/Mb) and therapeutic response [53-55]. Unlike cancers with germline or somatic defects that lead to genome instability and elevated mutation rates, these cancers develop due to exposure to environmental mutagens that result in increased TMB (UV radiation from sunlight and cigarette smoke). Importantly, the observed connection of TMB to immune checkpoint response led to the hypothesis that patients with MMRd cancers, indicated by MSI 'high' diagnoses, 
might also respond to these therapies. The definitive clinical trial of checkpoint blockade therapy in MMRd cancers was initially published in 2015 [56] and indicated a trend toward therapeutic response to pembrolizumab, an anti PD-1 therapy, in MSI high/MMRd cancers. The results of the phase 2 trial, in which patients diagnosed with MMR-deficient cancers in many different tissue sites were enrolled, were reported in 2017 [57]. This phase 2 trial provided the registration data that resulted in FDA approval of pembrolizumab for all cancers with a clinical diagnosis of MSI high cancer from any tissue site. Subsequent trials and manuscripts reporting the results of immune checkpoint blockade treatments in the context of different underlying types of genomic instability are summarized in Table 2.

Similarly, patients with advanced-stage melanoma have been treated in early-phase clinical trials of neoantigenbased vaccines (NCT00683670, NCT01970358, and NCT02035956), which used the genomic approaches outlined above to identify neoantigens. The neoantigens were utilized to construct patient-specific, multi-epitope vaccines using different vaccine platforms, including dendritic cell vaccines, long peptide vaccines, and RNA-encoded neoantigen vaccines. The three studies published to date have demonstrated that neoantigenspecific $\mathrm{T}$-cell populations were elicited in response to some of the vaccine-specified targets [67-69]. In two recent studies, patient responses were more durable when the vaccine was combined with an immune checkpoint blockade inhibitor $[68,69]$. A fourth study also has evaluated the neoantigen vaccine approach in adult patients with glioblastoma, demonstrating that patients who did not receive dexamethasone had increased infiltration of neoantigen-specific $\mathrm{T}$ cells into their tumors following vaccination [70].

\section{Genomic instability, neoantigens and immunotherapy response}

Modeling genomic instability in preclinical mouse models informs human cancer studies

Historically, cancer and cancer therapies have been studied preclinically in mouse models by introducing alterations in cancer-associated genes into the mouse genome, and then observing the development of cancer and its response to selected therapies. However, most genetically engineered mouse cancer models have a limitation in the context of neoantigens and immunotherapeutic response studies because their cancer genomes have few mutations. Thus, the cancers that are induced in these mouse models do not share the mutational burden seen in human cancers, including those impacted by genomic instability. One exception is the methylcholanthrene (MCA)-induced mouse sarcoma model that, similar to human melanomas, has an environmental contributor to its high mutational load. In this case, the mouse cancer was generated by treatment with the chemical carcinogen, MCA. Early studies of the MCA sarcoma model illustrated a high TMB, and in silico neoantigen prediction algorithms were able to identify neoantigenic peptides with strong MHC binding (relative to that of wild-type peptides), which resulted from amino acid sequence changes that were unique to the cancer. Further studies provided evidence of tumor elimination resulting from treatment of MCA sarcomas with a neoantigen-directed vaccine and immune checkpoint blockade inhibitors [51, 71]. The results from this carcinogen-induced cancer model reflect those from the human studies cited earlier with respect to the response of UV-associated melanomas to a combination of neoantigen vaccines and checkpoint blockade treatment. A more recent study from Schreiber's group builds upon these initial discoveries by focusing on the importance of MHCII restricted neoantigens in the vaccine-mediated immune

Table 2 Association of genome instability, alterations and immune therapy response

\begin{tabular}{|c|c|c|c|c|}
\hline Source of genome instability & Mutational profile/burden & Neoantigen load & Response to immunotherapy & References \\
\hline POLE mutation (germline or somatic) & $\begin{array}{l}\text { Single nucleotide variants } \\
\text { (SNVs)/ultra-hypermutated }\end{array}$ & High load & Checkpoint blockade responsive & {$[58,59]$} \\
\hline BRCA1/2 mutation (germline) & $\begin{array}{l}\text { Frameshift indels/elevated } \\
\text { proportion vs SNVs }\end{array}$ & $\begin{array}{l}\text { Medium load/elevated } \\
\text { number of strong binders }\end{array}$ & Checkpoint blockade responsive & [60] \\
\hline $\begin{array}{l}\text { Lynch syndrome (MSH1, MLH2, } \\
\text { MLH6, PMS2) }\end{array}$ & $\begin{array}{l}\text { SNVs and indels/ } \\
\text { hypermutated }\end{array}$ & $\begin{array}{l}\text { High load/elevated } \\
\text { number of strong binders }\end{array}$ & $\begin{array}{l}\text { Checkpoint blockade responsive, } \\
\text { personalized vaccine responsive }\end{array}$ & {$[57,61]$} \\
\hline VHL, SETD2, BAP1, KDM5C, FHIT defects & $\begin{array}{l}\text { Frameshift indels/elevated } \\
\text { proportion vs SNVs }\end{array}$ & $\begin{array}{l}\text { Medium load/elevated } \\
\text { number of strong binders }\end{array}$ & Checkpoint blockade responsive & {$[40,62,63$} \\
\hline $\begin{array}{l}\text { Xeroderma pigmentosum defect } \\
\text { (DDB2, ERCC1, ERCC2, ERCC3, } \\
\text { ERCC4, ERCC5, POLH, XPA, or XPC) }\end{array}$ & SNVs/ultra-hypermutated & High load & Checkpoint blockade responsive & [64] \\
\hline $\begin{array}{l}\text { Constitutional mismatch repair deficiency } \\
\text { syndrome (CMMRD): biallelic germline } \\
\text { mutations in Lynch syndrome genes } \\
\text { (MLH1, MSH2, MSH6, PMS2) }\end{array}$ & SNVs/hypermutated & High load & Checkpoint blockade responsive & {$[65,66]$} \\
\hline
\end{tabular}


response to cancers [72]. Here, $\mathrm{MHCI}$ and $\mathrm{MHCII}$ neoantigens from the MCA sarcoma model (mLAMA4 and mITGB1, respectively) were introduced either alone or in combination in an oncogene-driven sarcoma (KP) that lacks mutational neoantigens. Checkpoint blockade treatment in mice with contralateral tumors that expressed either both $\mathrm{MCHI}$ and MCHII neoantigens or only the $\mathrm{MHC} 1$ restricted neoantigen eliminated the former but not the latter. This result indicates that optimal anti-tumor responses to checkpoint blockade require the expression of both $\mathrm{MCHI}$ and $\mathrm{MCHI}$ neoantigens, which may have implications for human patients' responsiveness to immunotherapies.

Recently, an elegant study by Bardelli's group utilized transient Cas9 editing to knock out Mlh1, thereby inducing mismatch repair defects in mouse cancer cell lines [73]. This defect allowed the cell lines to grow into tumors in immunocompromised mice, and these tumors were subsequently transplanted into immunocompetent mice. The transplanted tumors were responsive to immune checkpoint blockade treatments, similar to human tumors exhibiting mismatch repair defects. Further genomic analysis of these MHL1-defective tumors as they grew over time in the immunocompetent mice demonstrated an increased and evolving neoantigen burden, indicating that DNA repair inactivation results in the continuous emergence of neoantigens in vivo. More recently, this group further investigated the longitudinal properties of neoantigen presentation by 45 colorectal cancer cell lines-including POLE-mutated, MSI-high, and microsatellite-stable examples propagated both in vitro (cell culture) and in vivo (xenografts)-and by patient-derived xenografts. Each example taken from the serial passage was evaluated by exome sequencing and RNAseq, with accompanying identification of single nucleotide variants (SNVs) and indels, as well as by neoantigen prediction. The results of this work illustrated that during cell-line growth in culture, in mouse xenografts or in patient-derived xenografts, MSI-high cells or cells with POLE mutations (with accompanying MSI-high genotypes) yielded an evolving neoantigen landscape over the longitudinal analysis. The MSI-high cells produced more frameshift indel neoantigens than did the POLE cells, which predominantly produce SNV neoantigens. RNA analysis of these samples illustrated that hypermutated colorectal cancer cells restrict host detection by selectively downregulating components of the neoantigen presentation process [74].

These results can be extrapolated to the human setting of mismatch repair defects, where cancers continue to occur over time with novel mutations and an accompanying high neoantigen burden [73]. A recent singlepatient study in the setting of a germline POLE defect parallels the results of Bardelli's group: comparisons of a primary glioblastoma to two spinal drop metastases (one prior to and one following checkpoint blockade inhibitor response) indicated an evolving neoantigen burden in each cancer sample studied [58]. Taken together, these results imply that checkpoint blockade therapies may have a protective or preventative efficacy in patients with underlying genomic instability resulting from MMRd, and encourages clinical trials to explore the use of these therapies in cancer prevention trials for patients who are highly likely to develop cancer.

\section{Pan-cancer evaluation of neoantigens and immunotherapy response}

Large-scale genomic studies of human cancers such as The Cancer Genome Atlas (TCGA) have provided the landscape of somatic and germline alterations, along with the transcriptome and methylome profiles, that largely define human cancers. More recently, computational approaches have emerged that are able to characterize the immune cell types that infiltrate tumors on the basis of the RNA sequencing data provided by studies such as TCGA. Using data available for the 20 solid cancer types included in TCGA, Trajanoski and colleagues recently published their computational evaluation of the pan-cancer immunogenome [75]. Here, the composition and functional orientation of the immune infiltrate, both cytotoxic and immunosuppressive, and the expression of neoantigenic peptides emerging from both somatic point mutations (SNVs) and cancer germline antigens were evaluated for 20 tumor types. The results of this study have been deposited into a web-accessible relational database called TCIA (https://tcia.at/). The findings have important implications that relate to the observation, across multiple studies of different tumor types and different immune checkpoint blockade therapies, that not all patients with an elevated tumor mutation burden, regardless of its origin, respond uniformly to this type of therapeutic intervention. In particular, Trajanoski and colleagues determined that, although elevated neoantigen burden resulting from increased mutational load had an impact on tumor immunogenicity, this was only one of several tumor-intrinsic factors that combined with tumorextrinsic factors (such as T-cell trafficking, the presence of immunomodulatory chemokines, and the infiltration of effector and immunosuppressive tumor-infiltrating lymphocytes) to determine the overall immunophenotype of a cancer [75]. These results invoke similar findings from studies of colorectal cancer immunity, including the impactful concept of 'Immunoscore' that emerged from Galon's group [76] and the subtype-specific nuances of immunogenicity in colorectal cancer patients with MSI and JAK1 mutations [77]. Immunoscore is based on the quantification of cytotoxic and memory $\mathrm{T}$ cells in the core of the tumor and its invasive margin, and has been shown 
to be a clinically useful prognostic marker. In the colorectal cancer study [77], the combination of specific gene expression subtyping (which yielded four consensus molecular subtypes) and genomic analysis (which identified the presence of loss-of-function JAK1 mutations), not neoantigen load, best predicted which MSI-high patients had the highest immune infiltration and best prognosis. In other words, predicting response to immune checkpoint blockade therapy is highly complex and requires the quantification of different variables that may be tissue-site specific.

A second pan-cancer study [40] explored focused insertion and deletion alterations (indels) and their contribution to the immunogenic phenotype. Here, cancers of 19 of the solid tumor types sequenced by TCGA were evaluated for predicted neoantigens on the basis of their exome sequencing data, as described earlier. In certain tumor types, data were available to evaluate associations between indel burden and treatment response for different immune checkpoint inhibitor therapies. This study revealed that renal cell carcinomas had more than double the median proportion of indels compared to all other cancer types, with an enrichment of high-affinity predicted neoantigens three times that of non-synonymous point mutations. The derived neoantigens were nine times enriched for specific binding compared to nonsynonymous point-mutation-encoded peptides. Correspondingly, the authors determined that responses to checkpoint blockade inhibitor therapies across three separate melanoma clinical trial cohorts $[55,78,79]$ were significantly associated with frameshift indel counts, which was a better predictor of response than were non-synonymous point mutation counts in two of the three studies.

\section{HRDs and immunotherapy response}

Several recent studies have further explored the relationship between genomic instability, immune cell infiltration, and, in some cases, response to immune checkpoint blockade in various tissue sites. One driver of such studies is the observation that not all patients with diagnosed MMRd cancers respond to these immunotherapies and, conversely, some patients with negative MMRd-assay results (based upon widely utilized diagnostic assays such as IHC and PCR-based MSI testing) do respond. The latter observation may be due to the type of assay used to diagnose MMRd cancers, as discussed below, because not all assays are equally sensitive. The former may be due to differences in the initiating genome alteration that drives mismatch repair defects, not all of which are equal in their impact, as also described below. Further, as explored above, other factors beyond the presence of mismatch repair defects determine treatment response or lack thereof.
In advanced prostate cancer, where two clinical trials testing response to immune checkpoint blockade in unselected patients have failed [80, 81], a report from de Bono and colleagues evaluated the diagnosis of MMRd using a variety of assays including IHC, MSI by PCR, MSI by targeted panel NGS of MMR pathway genes, and MSI by exome sequencing (WES) assay [82]. Their results showed that the PCR-based assay of MSI was more likely to give discordant (presumed false-positive) results when compared to the results of the NGS-based tests. This result indicates that not all assays for MSI detection and MMRd diagnosis are equal in diagnostic yield. Further, this study determined that prostate cancers with MMRd diagnosed by IHC or PCR-based MSI testing often, but not always, had corresponding higher mutational loads and MSI-positive results when tested by NGS. The associated analysis comparing immune cell infiltration via RNAseq deconvolution from 168 advanced prostate cancers in comparison to MMRd testing results demonstrated no positive association between total immune infiltrate and either overall mutation load (TMB) or MSI positivity as determined by targeted panel NGS assay, although MMRd mutational signature did correlate positively to higher inferred immune cell infiltration. Further analysis of mRNA expression for 762 immune-related genes in relation to MMRd status identified 24 genes whose expression was consistently correlated with MMRd diagnosis, and indicated that mismatch repair deficiencies associate with a more complex immune infiltrate, including the upregulation of genes associated with dendritic cells, macrophages, or myeloid cells and $\mathrm{T}$ cells. Taken together, this study indicates that a subset of lethal prostate cancers exhibit MMRd at diagnosis, that different assay methods can yield different diagnoses, and that only a proportion of diagnosed advanced prostate cancers have corresponding high TMB and stain with PD-L1 IHC. Hence, the sub-classification of advanced prostate cancer using NGS-based methods and evaluation of immune infiltration levels may better stratify patients who are likely to respond to immune checkpoint blockade treatments.

A separate study, which involved only immunohistochemistry-based analysis of endometrial cancers with a PD-L1 antibody, focused on comparing samples from patients with Lynch syndrome or $M L H 1$ promoter hypermethylation (MLH1hm) with MMRintact patient samples [83]. The PD-L1 expression results for LS, MLH1hm, and MMR-intact tumors indicated that the tumor cells in LS endometrial cancers had the highest expression of PD-L1, followed by MLH1hm and then MMR-intact samples. Hence, the potential benefit from PD-1 or PD-L1 therapy might vary depending on the molecular mechanism driving MMRd. 
Methylation-based silencing of homologous DNA recombination genes was recently reported in squamous cell histology cancers, including head and neck, cervical, and lung cancers [84]. Here, by extensively comparing the methylation in all homologous recombination genes to the IHC-based expression of CTLA-4 and PD-L1, the authors determined that squamous cell cancers hypermethylate $X R C C 3$ and $R A D 51 B$ and (in correlation) have elevated expression of the two immune checkpoint genes. Interestingly, the hypermethylation status of these DSB-repair genes (XRCC3 and RAD51B) led to elevated PD-L1 expression, a result that is discordant with the aforementioned result of a hypermethylated $M L H 1$ promoter in endometrial cancers by Sloan et al. [83]. On the basis of these two studies, it appears that the level of hypermethylation of different genes in mismatch and DSB-repair defects may be tissue-specific.

Mutation-driven genomic instability occurs in POLE- or POLD1-mutated cancers, where the levels of mutational burden (based on SNVs) in POLE exonuclease domain mutated cancers are extremely high. A recent study of the timing of POLE mutations established that these changes occur early in carcinogenesis and are detectable in preneoplastic lesions of both endometrial and colorectal cancers [85]. Correspondingly, evidence of CD8+ T-cell infiltration was also identified in the precursor lesions, lending credibility to the idea that these mutations occur early in the transition from normal to cancer cell, and that the neoantigens resulting from $P O L E$-driven genome instability recruit immune cells that predicate the high amount of immune cell infiltration observed in resected tumors of both endometrium and colon or rectum. These findings have potentially important implications for the treatment of patients diagnosed with POLE-mutated cancers and corresponding ultra-high mutation levels that may vary depending upon tissue site.

A similar premise of evaluating immune involvement during progression from normal cells to cancerous lesions was recently reported in squamous cell carcinoma of the lung (SCC). Here, Galon's group used gene expression data and multispectral imaging to characterize and compare biopsies representing nine stages of SCC development [86]. SCC is predominately a smoking-related cancer with a correspondingly high mutational load reflecting DNA damage from smoke carcinogens. Accordingly, this study characterized pre-neoplastic tissues as having the earliest molecular changes that activate immune sensing and response, whereas subsequent stages are distinguished by continual cell proliferation and accumulating somatic mutations that elicit an anti-tumor immune response. This in turn leads to high grade precancerous lesions with inherent immune suppression mechanisms just prior to progression to frank SCC. This study provides unique insights into early tumorimmune system interactions.
Collectively, these studies illustrate that not all genetic contributions to genome instability, to neoantigen generation, or to immune therapy responses are yet understood, and indeed that even when they are understood, these genetic contributions may not predict universal consequences for treatment outcomes.

\section{Implications for precision medicine}

The implications of the studies described above on the use of immune checkpoint blockade therapies in the context of precision cancer medicine for patients with genomic instability are significant. First, they imply that a tissue-specific set of diagnostic assays may be important for determining which patients are most likely to respond to these drugs, which are expensive and which have significant associated toxicities for certain patients. These assays will need to be devised and tested on retrospective samples from clinical trials of each drug in each tissue site (assuming those trials and samples exist), in order to demonstrate their predictive potential, before they can advance to use in a randomized clinical trial that would confirm their role as a companion diagnostic. Second, this scenario significantly adds to the complexity and time-to-result for cancer patients, and invokes a higher cost of diagnostic testing that may not be reimbursed by insurance companies or governments with socialized medicine programs. Indeed, because most metastatic cancer patients have received multiple lines of therapy prior to checkpoint blockade therapy, many of which suppress the immune system to different degrees, and because they also have variable levels of disease burden that probably impact their response, we may never be able to predict immune checkpoint blockade response fully in every patient by using the same set of assays. Nonetheless, the standardization of NGSbased assays and analytical pipelines for determining $\mathrm{TMB}$, neoantigen burden, and alterations to genes that impart genomic instability by studying both cancer and germline DNA is a worthwhile goal. In most cases, the same NGS data set can be used to evaluate the mutational status of important determinants of both immune status and neoantigen presentation, such as the mutational or gene-expression-based silencing of different $H L A$ alleles or of beta-2-microglobulin $\left(\beta_{2} \mathrm{M}\right)$, and of the activation of JAK/STAT pathways, which may also be indicative of existing or emergent resistance to checkpoint blockade therapy [87-91]. Specifying the optimal integration of diagnostic results from such NGS assays with those from conventional pathology-based assays (such as IHCstaining of CTLA-4, PD-1, and PD-L1 proteins) may drive a comprehensive evaluation of each patient that contributes to improved response prediction and may also indicate alternative therapeutic approaches when checkpoint blockade therapy is contraindicated. 


\section{Conclusions and future directions}

Genome instability in cancer results from a variety of genomic alterations, both germline and somatic. These alterations can be detected by different methods that reflect this variability in the underlying genes and their alterations, or can be simply evaluated by examining the downstream manifestation of the genomic defect using assays such as the detection of microsatellite instability. The recent studies reviewed here have begun to illustrate that not all types of genomic instability have the same impact when viewed in the context of immune cell recruitment or response to immune checkpoint blockade therapy. For example, even the widely accepted metric of $\mathrm{TMB}$ as a predictor of immune checkpoint blockade response is nuanced by other factors, both tumor intrinsic and extrinsic, that determine the likely response to immune modulatory drugs. Related to this conclusion, one pancancer study determined that frameshift alterations that result from insertion or deletion mutations produce strongly neoantigenic peptides and, overall, better predicted clinical responses to checkpoint blockade therapies [40].

Furthermore, there may be differences in mechanistic aspects of genomic instability that must be understood in the context of likely therapeutic response, such as the finding that cancers with hypermethylation-related MMRd appear to have reduced immune cell infiltration relative to mutation-related MMRd in several cancer types. These studies and others described in this review help to illustrate why TMB remains an imperfect predictor of therapeutic response to checkpoint blockade therapy as a standalone test across diverse tissue sites.

Evaluations of different methods to diagnose MSI-high cancers have demonstrated that NGS-based testing to detect microsatellite instability appears to be more sensitive than established methods such as PCR-based MSI assays. If designed correctly, NGS assays also can return information about resistance-associated alterations in immune response genes, overall TMB/neoantigen load, and different types of genomic alterations that may more accurately predict immunotherapy response. Driving the argument for the clinical benefit of such assays will require proper clinical trials that can ultimately provide a more confident prediction of response to expensive therapies and justify insurance reimbursement. Finally, several studies presented in this review emphasize that only the combination of correlative studies of banked tissues from clinical trials of different immune therapies, for which therapy response and outcomes are known, will enhance our understanding of the complex interplay of genomic instability, neoantigen generation, and immunomodulatory therapies. These studies, in turn, will inform the clinical management of cancer patients being treated with immunotherapy and will emphasize the gaps in our understanding of basic cancer immunity that require further elucidation.

\section{Abbreviations}

DSB: Double-stranded break; HLA: Human leukocyte antigen;

IHC: Immunohistochemistry; MCA: Methylcholanthrene; MHC: Major histocompatibility complex; MLH1 hm: MLH1 promoter hypermethylation; MMR: Mismatch repair; MMRd: Mismatch repair defect; MSI: Microsatellite instability; NGS: Next generation sequencing; SNV: Single nucleotide variant; TCGA: The Cancer Genome Atlas; TMB: Tumor mutational burden

\section{Acknowledgements}

The author wishes to acknowledge the collaboration and friendship of Dr. Robert D. Schreiber, who introduced her to the key concepts of cancer immunology and has provided valuable insights and mentorship ever since.

\section{Authors' contributions}

The author proposed, conceptualized, and wrote this review in its entirety, with acknowledgement of the referees who contributed to better organization and presentation of the materials covered. The author read and approved the final manuscript.

\section{Funding}

The author wishes to acknowledge the Nationwide Foundation Innovation Fund.

\section{Competing interests}

The author declares that she has no competing interests.

Received: 2 April 2019 Accepted: 5 November 2019

Published online: 20 November 2019

\section{References}

1. Kunkel TA. DNA replication fidelity. J Biol Chem. 2004;279:16895-8.

2. Arana ME, Kunkel TA. Mutator phenotypes due to DNA replication infidelity. Semin Cancer Biol. 2010;20:304-11.

3. Bebenek K, Kunkel TA. Functions of DNA polymerases. Adv Protein Chem. 2004;69:137-65.

4. Kane DP, Shcherbakova PV. A common cancer-associated DNA polymerase $\varepsilon$ mutation causes an exceptionally strong mutator phenotype, indicating fidelity defects distinct from loss of proofreading. Cancer Res. 2014;74:1895-901.

5. Jiricny J. The multifaceted mismatch-repair system. Nat Rev Mol Cell Biol. 2006;7:335-46.

6. Funkhouser WK Jr, Lubin IM, Monzon FA, Zehnbauer BA, Evans JP, Ogino S, et al. Relevance, pathogenesis, and testing algorithm for mismatch repairdefective colorectal carcinomas: a report of the association for molecular pathology. J Mol Diagn. 2012;14:91-103.

7. Kastrinos F, Syngal S. Inherited colorectal cancer syndromes. Cancer J. 2011; 17:405-15.

8. Wimmer K, Kratz CP. Constitutional mismatch repair-deficiency syndrome. Haematologica. 2010;95:699-701.

9. Win AK, Jenkins MA, Dowty JG, Antoniou AC, Lee A, Giles GG, et al. Prevalence and penetrance of major genes and polygenes for colorectal cancer. Cancer Epidemiol Biomark Prev. 2017;26:404-12.

10. Pearlman R, Frankel WL, Swanson B, Zhao W, Yilmaz A, Miller K, et al. Prevalence and spectrum of germline cancer susceptibility gene mutations among patients with early-onset colorectal cancer. JAMA Oncol. 2017;3:464-71.

11. Veigl ML, Kasturi L, Olechnowicz J, Ma AH, Lutterbaugh JD, Periyasamy S, et al. Biallelic inactivation of hMLH1 by epigenetic gene silencing, a novel mechanism causing human MSI cancers. Proc Natl Acad Sci U S A. 1998;95: 8698-702.

12. Wimmer K, Beilken A, Nustede R, Ripperger T, Lamottke B, Ure B, et al. A novel germline POLE mutation causes an early onset cancer prone syndrome mimicking constitutional mismatch repair deficiency. Familial Cancer. 2016;16:67-71.

13. Hansen MF, Johansen J, Bjørnevoll I, Sylvander AE, Steinsbekk KS, Sætrom P, et al. A novel POLE mutation associated with cancers of colon, pancreas, ovaries and small intestine. Familial Cancer. 2015;14:437-48.

14. Castellsagué E, Li R, Aligue R, González S, Sanz J, Martin E, et al. Novel POLE pathogenic germline variant in a family with multiple primary tumors results in distinct mutational signatures. Hum Mutat. 2019:40:36-41.

15. Billingsley CC, Cohn DE, Mutch DG, Stephens JA, Suarez AA, Goodfellow PJ. Polymerase $\varepsilon$ (POLE) mutations in endometrial cancer: clinical outcomes and implications for lynch syndrome testing. Cancer. 2014;121:386-94. 
16. Buchanan DD, Stewart JR, Clendenning M, Rosty C, Mahmood K, Pope BJ, et al. Risk of colorectal cancer for carriers of a germ-line mutation in POLE or POLD1. Genet Med. 2018;20:890-5.

17. Rosner G, Gluck N, Carmi S, Bercovich D, Fliss-Issakov N, Ben-Yehoyada M, et al. POLD1 and POLE gene mutations in Jewish cohorts of early-onset colorectal cancer and of multiple colorectal adenomas. Dis Colon Rectum. 2018;61:1073-9.

18. Esteban-Jurado C, Giménez-Zaragoza D, Muñoz J, Franch-Expósito S Álvarez-Barona M, Ocaña T, et al. POLE and POLD1 screening in 155 patients with multiple polyps and early-onset colorectal cancer. Oncotarget. 2017;8:26732-43.

19. Ferrer-Avargues R, Díez-Obrero V, Martín-Tomás E, Hernández-Illán E, Castillejo M-I, Codoñer-Alejos A, et al. Characterization of a novel POLD1 missense founder mutation in a Spanish population. J Gene Med. 2017;19. https://doi.org/10.1002/jgm.2951.

20. King M-C, Marks JH, Mandell JB, New York Breast Cancer Study Group. Breast and ovarian cancer risks due to inherited mutations in BRCA1 and BRCA2. Science. 2003;302:643-6.

21. Rahman N, Seal S, Thompson D, Kelly P, Renwick A, Elliott A, et al. PALB2, which encodes a BRCA2-interacting protein, is a breast cancer susceptibility gene. Nat Genet. 2007;39:165-7.

22. Connor F, Smith A, Wooster R, Stratton M, Dixon A, Campbell E, et al. Cloning, chromosomal mapping and expression pattern of the mouse Brca2 gene. Hum Mol Genet. 1997;6:291-300.

23. McBride DJ, Etemadmoghadam D, Cooke SL, Alsop K, George J, Butler A, et al. Tandem duplication of chromosomal segments is common in ovarian and breast cancer genomes. J Pathol. 2012;227:446-55.

24. Offit $K$, Thom P. Ethical and legal aspects of cancer genetic testing. Semin Oncol. 2007;34:435-43.

25. Werness BA, Ramus SJ, Whittemore AS, Garlinghouse-Jones K, Oakley-Girvan I, Dicioccio RA, et al. Histopathology of familial ovarian tumors in women from families with and without germline BRCA1 mutations. Hum Pathol. 2000;31:1420-4.

26. Stadler ZK, Battaglin F, Middha S, Hechtman JF, Tran C, Cercek A, et al. Reliable detection of mismatch repair deficiency in colorectal cancers using mutational load in next-generation sequencing panels. J Clin Oncol. 2016; 34:2141-7.

27. Alexandrov LB, Nik-Zainal S, Wedge DC, Aparicio SAJR, Behjati S, Biankin AV, et al. Signatures of mutational processes in human cancer. Nature. 2013;500: 415-21.

28. Cortes-Ciriano I, Lee S, Park W-Y, Kim T-M, Park PJ. A molecular portrait of microsatellite instability across multiple cancers. Nat Commun. 2017:8:15180.

29. Davies H, Glodzik D, Morganella S, Yates LR, Staaf J, Zou X, et al. HRDetect is a predictor of BRCA1 and BRCA2 deficiency based on mutational signatures. Nat Med. 2017;23:517-25.

30. Chalmers ZR, Connelly CF, Fabrizio D, Gay L, Ali SM, Ennis R, et al. Analysis of 100,000 human cancer genomes reveals the landscape of tumor mutational burden. Genome Med. 2017:9:34

31. Berger MF, Mardis ER. The emerging clinical relevance of genomics in cancer medicine. Nat Rev Clin Oncol. 2018;15:353-65.

32. Szolek A, Schubert B, Mohr C, Sturm M, Feldhahn M, Kohlbacher O. OptiType: precision HLA typing from next-generation sequencing data. Bioinformatics. 2014;30:3310-6.

33. Shukla SA, Rooney MS, Rajasagi M, Tiao G, Dixon PM, Lawrence MS, et al. Comprehensive analysis of cancer-associated somatic mutations in class I HLA genes. Nat Biotechnol. 2015;33:1152-8.

34. Hundal J, Miller CA, Griffith M, Griffith OL, Walker J, Kiwala S, et al. Cancer immunogenomics: computational neoantigen identification and vaccine design. Cold Spring Harb Symp Quant Biol. 2016;81:105-11.

35. Hundal J, Carreno BM, Petti AA, Linette GP, Griffith OL, Mardis ER, et al pVAC-Seq: a genome-guided in silico approach to identifying tumor neoantigens. Genome Med. 2016:8:11.

36. O'Donnell TJ, Rubinsteyn A, Bonsack M, Riemer AB, Laserson U, Hammerbacher J. MHCflurry: open-source class I MHC binding affinity prediction. Cell Syst. 2018;7:129-32.

37. Rubinsteyn A, Hodes I, Kodysh J, Hammerbacher J. Vaxrank: a computational tool for designing personalized cancer vaccines. bioRxiv. 2017. https://doi. org/10.1101/142919.

38. Richters MM, Xia H, Campbell KM, Gillanders WE, Griffith OL, Griffith M. Best practices for bioinformatic characterization of neoantigens for clinical utility. Genome Med. 2019;11:56.
39. Gubin MM, Artyomov MN, Mardis ER, Schreiber RD. Tumor neoantigens: building a framework for personalized cancer immunotherapy. J Clin Invest. 2015;125:3413-21.

40. Turajlic S, Litchfield K, Xu H, Rosenthal R, McGranahan N, Reading JL, et al. Insertion-and-deletion-derived tumour-specific neoantigens and the immunogenic phenotype: a pan-cancer analysis. Lancet Oncol. 2017;18: 1009-21.

41. Linnebacher M, Gebert J, Rudy W, Woerner S, Yuan YP, Bork P, et al. Frameshift peptide-derived T-cell epitopes: a source of novel tumor-specific antigens. Int J Cancer. 2001;93:6-11.

42. Cai A, Keskin DB, DeLuca DS, Alonso A, Zhang W, Zhang GL, et al. Mutated BCR-ABL generates immunogenic T-cell epitopes in CML patients. Clin Cancer Res. 2012:18:5761-72.

43. Yang W, Lee K-W, Srivastava RM, Kuo F, Krishna C, Chowell D, et al. Immunogenic neoantigens derived from gene fusions stimulate $T$ cell responses. Nat Med. 2019;25:767-75.

44. Zamora AE, Crawford JC, Allen EK, Guo X-ZJ, Bakke J, Carter RA, et al. Pediatric patients with acute lymphoblastic leukemia generate abundant and functional neoantigen-specific CD8 T cell responses. Sci Transl Med. 2019;11. https://doi.org/10.1126/scitrans/med.aat8549.

45. Laumont CM, Vincent K, Hesnard L, Audemard É, Bonneil É, Laverdure J-P, et al. Noncoding regions are the main source of targetable tumor-specific antigens. Sci Transl Med. 2018;10. https://doi.org/10.1126/scitrans/med.aau5516.

46. Bulik-Sullivan B, Busby J, Palmer CD, Davis MJ, Murphy T, Clark A, et al. Deep learning using tumor HLA peptide mass spectrometry datasets improves neoantigen identification. Nat Biotechnol. 2018. https:/doi.org/10.1038/nbt.4313.

47. Bassani-Sternberg M. Mass spectrometry based immunopeptidomics for the discovery of cancer neoantigens. Methods Mol Biol. 2018;1719:209-21.

48. Kalaora S, Wolf Y, Feferman T, Barnea E, Greenstein E, Reshef D, et al. Combined analysis of antigen presentation and T-cell recognition reveals restricted immune responses in melanoma. Cancer Discov. 2018;8:1366-75.

49. Leach DR, Krummel MF, Allison JP. Enhancement of antitumor immunity by CTLA-4 blockade. Science. 1996;271:1734-6.

50. Freeman GJ, Long AJ, Iwai Y, Bourque K, Chernova T, Nishimura $H$, et al. Engagement of the PD-1 immunoinhibitory receptor by a novel $B 7$ family member leads to negative regulation of lymphocyte activation. J Exp Med. 2000;192:1027-34

51. Gubin MM, Zhang $X$, Schuster $H$, Caron E, Ward JP, Noguchi $T$, et al. Checkpoint blockade cancer immunotherapy targets tumour-specific mutant antigens. Nature. 2014;515:577-81.

52. Sharma P, Allison JP. The future of immune checkpoint therapy. Science. 2015;348:56-61.

53. McGranahan N, Furness AJS, Rosenthal R, Ramskov S, Lyngaa R, Saini SK et al. Clonal neoantigens elicit T cell immunoreactivity and sensitivity to immune checkpoint blockade. Science. 2016;351:1463-9.

54. Rizvi NA, Hellmann MD, Snyder A, Kvistborg P, Makarov V, Havel JJ, et al. Cancer immunology. Mutational landscape determines sensitivity to PD-1 blockade in non-small cell lung cancer. Science. 2015;348:124-8.

55. Snyder A, Makarov V, Merghoub T, Yuan J, Zaretsky JM, Desrichard A, et al. Genetic basis for clinical response to CTLA-4 blockade in melanoma. N Engl J Med. 2014;371:2189-99.

56. Le DT, Uram JN, Wang H, Bartlett BR, Kemberling H, Eyring AD, et al. PD-1 blockade in tumors with mismatch-repair deficiency. N Engl J Med. 2015; 372:2509-20.

57. Le DT, Durham JN, Smith KN, Wang H, Bartlett BR, Aulakh LK, et al. Mismatch repair deficiency predicts response of solid tumors to PD-1 blockade. Science. 2017;357:409-13.

58. Johanns TM, Miller CA, Dorward IG, Tsien C, Chang E, Perry A, et al. Immunogenomics of hypermutated glioblastoma: a patient with germline POLE deficiency treated with checkpoint blockade immunotherapy. Cancer Discov. 2016:6:1230-6.

59. Henninger EE, Pursell ZF. DNA polymerase $\varepsilon$ and its roles in genome stability. IUBMB Life. 2014;66:339-51.

60. Kraya AA, Maxwell KN, Wubbenhorst B, Wenz BM, Pluta J, Rech AJ, et al. Genomic signatures predict the immunogenicity of BRCA-deficient breast cancer. Clin Cancer Res. 2019;25:4363-74.

61. Schwitalle Y, Kloor M, Eiermann S, Linnebacher M, Kienle P, Knaebel HP, et al. Immune response against frameshift-induced neopeptides in HNPCC patients and healthy HNPCC mutation carriers. Gastroenterology. 2008;134:988-97.

62. Ruiz-Bañobre J, Anido U, Abdulkader I, Antúnez-López J, López-López R, García-González J. Long-term response to nivolumab and acute renal failure 
in a patient with metastatic papillary renal cell carcinoma and a PD-L1 tumor expression increased with sunitinib therapy: a case report. Front Oncol. 2016;6:250.

63. Geynisman DM. Anti-programmed cell death protein 1 (PD-1) antibody nivolumab leads to a dramatic and rapid response in papillary renal cell carcinoma with sarcomatoid and rhabdoid features. Eur Urol. 2015;68:912-4.

64. Momen S, Fassihi H, Davies HR, Nikolaou C, Degasperi A, Stefanato CM, et al. Dramatic response of metastatic cutaneous angiosarcoma to an immune checkpoint inhibitor in a patient with xeroderma pigmentosum: whole-genome sequencing aids treatment decision in end-stage disease. Cold Spring Harb Mol Case Stud. 2019;5(5):a004408.

65. Bouffet E, Larouche V, Campbell BB, Merico D, de Borja R, Aronson M, et al. Immune checkpoint inhibition for hypermutant glioblastoma multiforme resulting from germline biallelic mismatch repair deficiency. J Clin Oncol. 2016:34:2206-11.

66. Shlien A, Campbell BB, de Borja R, Alexandrov LB, Merico D, Wedge D, et al. Combined hereditary and somatic mutations of replication error repair genes result in rapid onset of ultra-hypermutated cancers. Nat Genet. 2015; 47:257-62.

67. Carreno BM, Magrini V, Becker-Hapak M, Kaabinejadian S, Hundal J, Petti AA, et al. Cancer immunotherapy. A dendritic cell vaccine increases the breadth and diversity of melanoma neoantigen-specific T cells. Science. 2015;348: 803-8.

68. Ott PA, Hu Z, Keskin DB, Shukla SA, Sun J, Bozym DJ, et al. An immunogenic personal neoantigen vaccine for patients with melanoma. Nature. 2017;547: 217-21.

69. Sahin U, Derhovanessian E, Miller M, Kloke B-P, Simon P, Löwer M, et al. Personalized RNA mutanome vaccines mobilize poly-specific therapeutic immunity against cancer. Nature. 2017;547:222-6.

70. Keskin DB, Anandappa AJ, Sun J, Tirosh I, Mathewson ND, Li S, et al. Neoantigen vaccine generates intratumoral $T$ cell responses in phase $\mathrm{lb}$ glioblastoma trial. Nature. 2019:565:234-9.

71. Matsushita H, Vesely MD, Koboldt DC, Rickert CG, Uppaluri R, Magrini VJ, et al. Cancer exome analysis reveals a T-cell-dependent mechanism of cancer immunoediting. Nature. 2012;482:400-4.

72. Alspach E, Lussier DM, Miceli AP, Kizhvatov I, DuPage M, Luoma AM, et al. MHC-II neoantigens shape tumour immunity and response to immunotherapy. Nature. 2019. https://doi.org/10.1038/s41586-019-1671-8.

73. Germano G, Lamba S, Rospo G, Barault L, Magrì A, Maione F, et al. Inactivation of DNA repair triggers neoantigen generation and impairs tumour growth. Nature. 2017;552:116-20.

74. Rospo G, Lorenzato A, Amirouchene-Angelozzi N, Magrì A, Cancelliere C, Corti $\mathrm{G}$, et al. Evolving neoantigen profiles in colorectal cancers with DNA repair defects. Genome Med. 2019:11:42.

75. Charoentong P, Finotello F, Angelova M, Mayer C, Efremova M, Rieder D, et al. Pan-cancer immunogenomic analyses reveal genotypeimmunophenotype relationships and predictors of response to checkpoint blockade. Cell Rep. 2017;18:248-62.

76. Mlecnik B, Bindea G, Angell HK, Maby P, Angelova M, Tougeron D, et al. Integrative analyses of colorectal cancer show immunoscore is a stronger predictor of patient survival than microsatellite instability. Immunity. 2016; 44:698-711.

77. Sveen A, Johannessen B, Tengs T, Danielsen SA, Eilertsen IA, Lind GE, et al. Multilevel genomics of colorectal cancers with microsatellite instabilityclinical impact of JAK1 mutations and consensus molecular subtype 1. Genome Med. 2017:9:46

78. Van Allen EM, Miao D, Schilling B, Shukla SA, Blank C, Zimmer L, et al. Genomic correlates of response to CTLA-4 blockade in metastatic melanoma. Science. 2015;350:207-11.

79. Hugo W, Zaretsky JM, Sun L, Song C, Moreno BH, Hu-Lieskovan S, et al. Genomic and transcriptomic features of response to anti-PD-1 therapy in metastatic melanoma. Cell. 2017;168:542.

80. Kwon ED, Drake CG, Scher HI, Fizazi K, Bossi A, van den Eertwegh AJM, et al. Ipilimumab versus placebo after radiotherapy in patients with metastatic castration-resistant prostate cancer that had progressed after docetaxel chemotherapy (CA184-043): a multicentre, randomised, double-blind, phase 3 trial. Lancet Oncol. 2014;15:700-12.

81. Beer TM, Kwon ED, Drake CG, Fizazi K, Logothetis C, Gravis G, et al. Randomized, double-blind, phase III trial of ipilimumab versus placebo in asymptomatic or minimally symptomatic patients with metastatic chemotherapy-naive castration-resistant prostate cancer. J Clin Oncol. 2017;35:40-7.
82. Rodrigues DN, Rescigno P, Liu D, Yuan W, Carreira S, Lambros MB, et al. Immunogenomic analyses associate immunological alterations with mismatch repair defects in prostate cancer. J Clin Invest. 2018;128:5185.

83. Sloan EA, Ring KL, Willis BC, Modesitt SC, Mills AM. PD-L1 expression in mismatch repair-deficient endometrial carcinomas, including lynch syndrome-associated and MLH1 promoter hypermethylated tumors. Am J Surg Pathol. 2017;41:326-33.

84. Rieke DT, Ochsenreither S, Klinghammer K, Seiwert TY, Klauschen F, Tinhofer I, et al. Methylation of RAD51B, XRCC3 and other homologous recombination genes is associated with expression of immune checkpoints and an inflammatory signature in squamous cell carcinoma of the head and neck, lung and cervix. Oncotarget. 2016;7:75379-93.

85. Temko D, Van Gool IC, Rayner E, Glaire M, Makino S, Brown M, et al. Somatic POLE exonuclease domain mutations are early events in sporadic endometrial and colorectal carcinogenesis, determining driver mutational landscape, clonal neoantigen burden and immune response. J Pathol. 2018; 245:283-96.

86. Mascaux C, Angelova M, Vasaturo A, Beane J, Hijazi K, Anthoine G, et al. Immune evasion before tumour invasion in early lung squamous carcinogenesis. Nature. 2019;571:570-5.

87. Zaretsky JM, Garcia-Diaz A, Shin DS, Escuin-Ordinas H, Hugo W, HuLieskovan S, et al. Mutations associated with acquired resistance to PD-1 blockade in melanoma. N Engl J Med. 2016;375:819-29.

88. Budczies J, Bockmayr M, Klauschen F, Endris V, Fröhling S, Schirmacher $P$, et al. Mutation patterns in genes encoding interferon signaling and antigen presentation: a pan-cancer survey with implications for the use of immune checkpoint inhibitors. Genes Chromosomes Cancer. 2017;56:651-9.

89. Yeon S, Jung S-H, Jo YS, Choi EJ, Kim MS, Chung Y-J, et al. Immune checkpoint blockade resistance-related B2M hotspot mutations in microsatellite-unstable colorectal carcinoma. Pathol Res Pract. 2019;215:209-14.

90. Sade-Feldman M, Jiao YJ, Chen JH, Rooney MS, Barzily-Rokni M, Eliane J-P, et al. Resistance to checkpoint blockade therapy through inactivation of antigen presentation. Nat Commun. 2017:8:1136.

91. Rosenthal R, Cadieux EL, Salgado R, Bakir MA, Moore DA, Hiley CT, et al. Neoantigen-directed immune escape in lung cancer evolution. Nature. 2019;567:479-85.

\section{Publisher's Note}

Springer Nature remains neutral with regard to jurisdictional claims in published maps and institutional affiliations. 